\title{
Heiße Getränke erhöhen das Ösophaguskarzinomrisiko
}

\begin{abstract}
Wenn ein Zusammenhang zwischen dem regelmäßigen Genuss eines warmen Getränks und dem Ösophaguskarzinom untersucht wird, muss die Temperatur des Getränks ebenso berücksichtigt werden wie die konsumierte Menge; denn das Risiko kann sowohl durch Hitzeschäden als auch durch die chemische Zusammensetzung des Getränks beeinflusst werden.
\end{abstract}

- Dieser komplexen Fragestellung wurde im Rahmen einer epidemiologischen Studie nachgegangen. Analysiert wurden die Auswirkungen von Kaffee, Tee und Matetee, wobei Menge und Temperatur getrennt berücksichtigt wurden. Insgesamt wurden im Rahmen dieser Metaanalyse die Ergebnisse von 59 Studien ausgewertet.

Für Kaffee und Tee ergaben sich kaum Hinweise für eine Assoziation zwischen der Menge und dem Ösophaguskarzinom. Doch in der Mehrzahl der Studien fand sich ein signifikant erhöhtes Ösophaguskarzinomrisiko bei regelmäßigem Genuss heißer Getränke. Für Matetee gibt es nur wenige Studien, die jedoch ein erhöhtes Karzinomrisiko in Abhängigkeit sowohl von der Menge als auch von der Temperatur signalisieren.

\section{r-}

\section{-}

KOMMENTAR: Diese Studie dürfte sowohl die Kaffee- als auch die Teetrinker beruhigen, denn es gibt bisher keinerlei wissenschaftlichen Beleg dafür, dass die Menge dieser Getränke das Ösophaguskarzinomrisiko erhöht. Doch grundsätzlich gilt für jede Form der Nahrungsaufnahme die Empfehlung, ,Heißes“ zu vermeiden; denn eine chronische Hitzeschädigung kann den Nährboden für eine maligne Entartung bereiten.

P. StiefelhageN - F. Islami et al.
High-temperature beverages and foods and
esophageal cancer risk - a systematic review. Int. J. Cancer 125(2009) 491-524
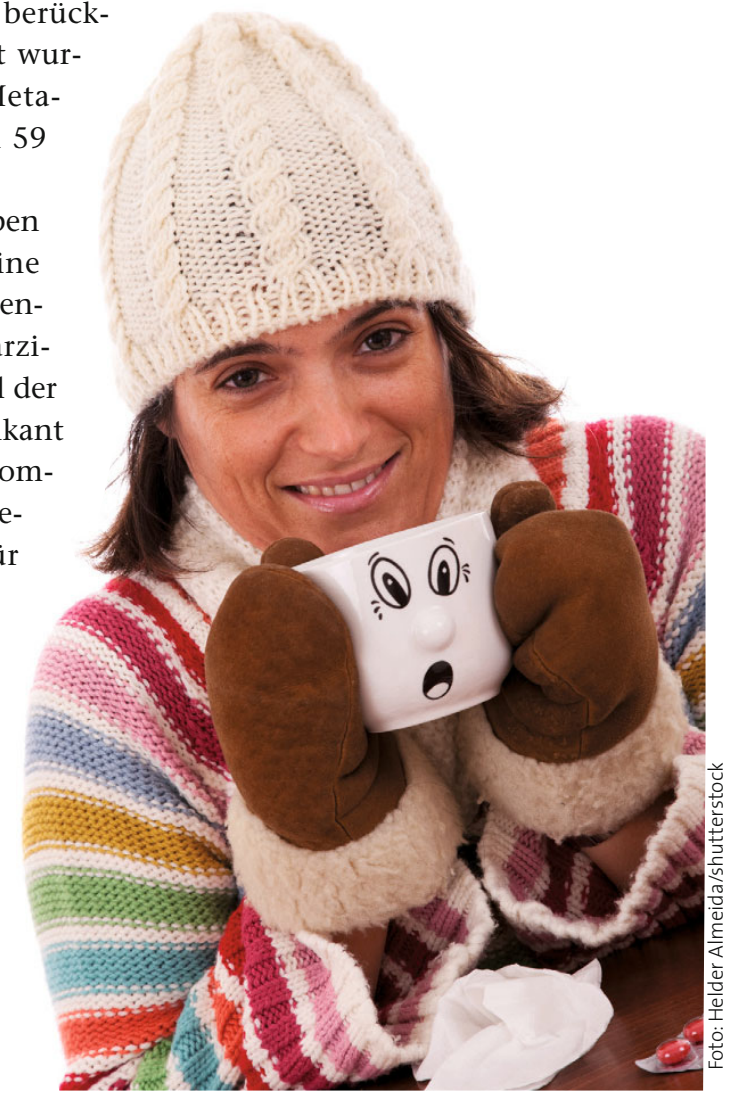

Heiße Getränke - für die Speiseröhre keine Wohltat.

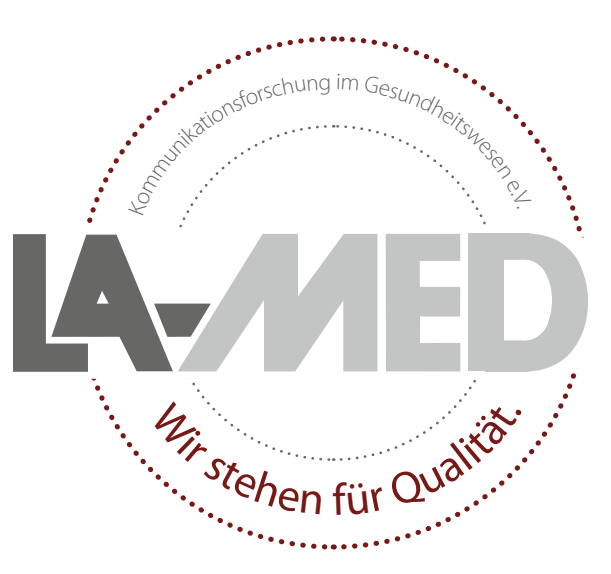

\section{Sie lesen Qualität}

Damit das auch so bleibt, befragen wir Sie in Kooperation mit tns sinfratest in den nächsten Wochen.

\section{Ihr Urteil ist uns wichtig.}

\section{Bitte nehmen Sie teil!}

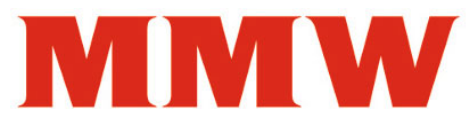

Fortschritte der Medizin
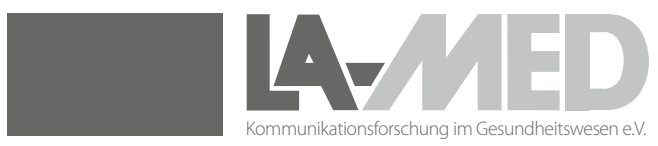